\title{
$\mathrm{Ag} @ \mathrm{SiO}_{2} @ \mathrm{GdF}_{3}: \mathrm{Yb}, \mathrm{Er}$ 核壳结构纳米材料的制备与增强上转换荧光
}

\author{
魏忠杰刘桂霞* 董相廷王进贤 于文生 \\ (长春理工大学化学与环境工程学院 应用化学与纳米技术吉林省高校重点实验室 长春 130022)
}

\begin{abstract}
摘要 采用直接沉淀法成功制备了 $\mathrm{Ag} @ \mathrm{SiO}_{2} @ \mathrm{GdF}_{3}: \mathrm{Er}, \mathrm{Yb}$ 核壳结构纳米上转换发光粒子, 并用 XRD, TEM, UV-Vis, FTIR 以及荧光光谱等对其结构和发光性能进行了表征. XRD 分析表明: Ag 表面包覆上了结晶良好的正交晶系的 $\mathrm{GdF}_{3}: \mathrm{Er}, \mathrm{Yb}$. TEM 照片显示: 制备的复合纳米粒子具有明显的球形核壳结构，内核 $\mathrm{Ag}$ 粒子的直径约 $50 \mathrm{~nm}$ 左右，包覆 后的 $\mathrm{Ag} @ \mathrm{SiO}_{2} @ \mathrm{GdF}_{3}: \mathrm{Er}, \mathrm{Yb}$ 粒径约为 $80 \sim 120 \mathrm{~nm}$, 表面光滑且包覆完全. UV-Vis 光谱证明: $\mathrm{GdF}_{3}: \mathrm{Er}, \mathrm{Yb}$ 和 $\mathrm{SiO}_{2}$ 成功包 覆在 $\mathrm{Ag}$ 核表面, 包覆后 $\mathrm{Ag}$ 纳米粒子的表面等离子体共振吸收峰发生了红移. 苂光光谱表明: 在 $980 \mathrm{~nm}$ 激光激发下, 该复合纳米粒子显示出和纯的 $\mathrm{GdF}_{3}: \mathrm{Er}, \mathrm{Yb}$ 相同的 $\mathrm{Er}^{3+}$ 的特征红色和绿色上转换发光, 以位于 $655 \mathrm{~nm}$ 处的 $\mathrm{Er}^{3+}$ 离子的 ${ }^{4} \mathrm{~F}_{9 / 2} \rightarrow{ }^{4} \mathrm{I}_{15 / 2}$ 的红光发射最强, 并且复合粒子的发射光强度比纯的 $\mathrm{GdF}_{3}: \mathrm{Er}$, $\mathrm{Yb}$ 有所增强.
\end{abstract}

关键词 $\mathrm{GdF}_{3}: \mathrm{Yb}, \mathrm{Er} ;$ 核壳结构; 上转换荧光

\section{Preparation of $\mathrm{Ag} @ \mathrm{SiO}_{2} @ \mathrm{GdF}_{3}: \mathrm{Er}, \mathrm{Yb}$ Core-shell Structure Nanomaterials and Enhanced Up-conversion Luminescence}

\author{
Wei, Zhongjie $\quad$ Liu, Guixia* Dong, Xiangting Wang, Jinxian $\quad$ Yu, Wensheng \\ (School of Chemistry and Environmental Engineering, Changchun University of Science and Technology, Key Labora- \\ tory of Applied Chemistry and Nanotechnology at Universities of Jilin Province, Changchun 130022)
}

\begin{abstract}
Noble metal nanoparticles such as $\mathrm{Ag}$ or $\mathrm{Au}$ and rare earth ions doped-up-conversion luminescence materials are all potential in the fields of biological labeling and sensing, diagnosis and biotherapy. When coupling with them together to form core-shell structure composites, noble metal nanostructures will present the strong surface plasmon resonances (SPRs), they can act as the function of "antenna", that is they can transfer the absorption energy to the luminescence particles and enhance their luminescence intensities. Moreover, when positioned in close proximity to metal surfaces, the luminescence materials can exhibit optical property changes (quenching or enhancement of luminescence), likely a result of the changed near-field electro-dynamical environment around the metal that arises from the collective oscillation of conduction electrons. This is a perfect model for studing the effects on luminescence properies of metal and luminescence materials. In this article, $\mathrm{Ag}$ nanoparticles were prepared by a glycol reduction method, and $\mathrm{SiO}_{2}$ spacers were coating on the surface of Ag nanoparticles by modified Stöber method, at last, the core-shell structure $\mathrm{Ag} @ \mathrm{SiO}_{2} @ \mathrm{GdF}_{3}: \mathrm{Er}, \mathrm{Yb}$ up-conversion luminescent nanoparticles were successfully synthesized by direct precipitation method. The structure and luminescence property of the samples were characterized by XRD, TEM, FTIR, UV-Vis and fluorescence spectra. XRD patterns show that the orthogonal phase $\mathrm{GdF}_{3}: \mathrm{Er}, \mathrm{Yb}$ nanocrystals are coated on the surface of Ag cores. TEM images present that the obtained composites have obvious spherical core-shell structure, the diameter of the Ag core is $50 \mathrm{~nm}$, the size of the $\mathrm{Ag} @ \mathrm{SiO}_{2} @ \mathrm{GdF}_{3}: \mathrm{Er}, \mathrm{Yb}$ composites is about $80 \sim 120 \mathrm{~nm}$, the surface is smooth and the coating is complete, $\mathrm{GdF}_{3}: \mathrm{Er}, \mathrm{Yb}$ nanoparticles are found obviously in the shell. UV-Vis spectra indicate that $\mathrm{GdF}_{3}: \mathrm{Er}, \mathrm{Yb}$ and $\mathrm{SiO}_{2}$ are coated successfully on the surface of $\mathrm{Ag}$, which increased the refractive index of local area around Ag nanoparticles, and the surface plasma absorption peaks of Ag have red shift. The up-conversion luminescent spectra also indicate that the core-shell structure composite particles have the same red and green up-conversion emissions as the pure $\mathrm{GdF}_{3}: \mathrm{Er}, \mathrm{Yb}$, their strong peaks are all near $655 \mathrm{~nm}$ corresponding to the ${ }^{4} \mathrm{~F}_{9 / 2} \rightarrow{ }^{4} \mathrm{I}_{15 / 2}$ transition of $\mathrm{Er}^{3+}$. The fluorescent intensity of the core-shell structure composites is stronger than that of the pure $\mathrm{GdF} \mathrm{F}_{3}: \mathrm{Er}, \mathrm{Yb}$ when the $\mathrm{SiO}_{2}$ as spacers, which indicates that $\mathrm{Ag}$ cores realize the up-conversion fluorescent enhancement of $\mathrm{GdF}$ : $\mathrm{Er}, \mathrm{Yb}$. The luminescence intensity is enhanced with the increasing of silica thickness and the amount of $\mathrm{GdF}_{3}: \mathrm{Er}, \mathrm{Yb}$. This core-shell structure has a certain reference value for preparation of highly efficient rare earth up-conversion luminescent nanomaterials. Keywords $\mathrm{GdF}_{3}: \mathrm{Yb}$,Er; core-shell structure; up-conversion luminescence
\end{abstract}

* E-mail: liuguixia22@yahoo.com.cn; Tel.: 0431-85582574; Fax: 0431-85583085

Received November 8, 2013; published November 26, 2013.

Project supported by the National Natural Science Foundation of China (No. 51072026) and the Development of Science and Technology Plan Projects of Jilin Province (No. 20130206002GX).

项目受国家自然科学基金面上项目(No. 51072026)和吉林省科技发展计划项目(No. 20130206002GX)资助. 


\section{1 引言}

纳米发光材料除了广泛被应用于照明、显示、激光 和 X 射线成像外, 在苂光标记、生物组织成像与识别以 及医学诊断与治疗等领域崭露头角, 逐渐在国际纳米材 料研究中占据十分重要的地位 ${ }^{[1 \sim 9]}$. 其中, 稀土掺杂上 转换纳米发光材料是一类重要的发光材料. 与传统的荧 光标记物(如有机染料、量子点等)相比较, 上转换纳米 发光粒子具有稳定性好、毒性低、发光强度高、Stokes 位移大等优点. 另外, 上转换纳米发光粒子的激发光为 红外光, 红外光对生物组织活体有良好的穿透能力, 对 生物样品造成光损伤比较小, 还可以有效避免生物体自 发荧光和散射光的干扰, 从而提高检测的灵敏度及信噪 比, 因此在生物标记和生物检测等领域有巨大的应用潜 力 $^{[10 \sim 15]}$. 如何更有效地提高发光材料的发光效率仍然 是科研工作者一直努力的方向. 贵金属纳米材料由于其 独特的等离子体共振效应及表面增强拉曼效应等卓越 的光学效应而备受关注. 其与发光基团复合形成核壳结 构复合材料以及其本身特性对发光基团发光性能的影 响的研究是目前国内外研究热点 ${ }^{[16 ~ 18]}$. 金属银纳米粒 子由于具有特殊的表面等离子体共振, 可极大地增强粒 子表面的局域电磁场, 改变吸附在颗粒表面及周围的苂 光团的自由空间条件, 从而改变苂光体的光谱. 在生物 医学领域, 包括疾病诊断、治疗、新型药物传递系统、 外科设备/移植以及 DNA 序列测定、免疫分析、生物检 验等生物技术领域中的应用展现出巨大的发展潜 力 $^{[19,20] \text {. }}$

目前, 关于贵金属和发光材料复合后使其发光增强 的报道己有很多. 如 Aslan 等 ${ }^{[21]}$ 在对比了 Eu-TDPA-掺 杂的 $\mathrm{Ag} @ \mathrm{SiO}_{2}$ 与 $\mathrm{Eu}-\mathrm{TDPA}$-掺杂的 $\mathrm{SiO}_{2}$ 和 $\mathrm{Rh} 800$-掺杂 的 $\mathrm{Ag} @ \mathrm{SiO}_{2}$ 与 $\mathrm{Rh} 800$-掺杂的 $\mathrm{SiO}_{2}$ 的荧光发射强度后 发现: $\mathrm{Ag}$ 的存在使其荧光发射强度分别增强了约 8 倍和 20 倍, 很好地证明了金属增强荧光理论. Min 等 ${ }^{[22]}$ 通过 两步两层包覆和煅烧过程制备出 $\mathrm{Au} @ \mathrm{Y}_{2} \mathrm{O}_{3}: \mathrm{Eu}^{3+}$ 核壳型 纳米粒子, 结果表明, $\mathrm{Au}$ 核有助于稀土离子的电子跃迁, 大大增强了 $\mathrm{Y}_{2} \mathrm{O}_{3}: \mathrm{Eu}^{3+}$ 的发光强度. Feng 等 ${ }^{[23]}$ 利用溶剂 蒸发法制备了 $\mathrm{Ag}-\mathrm{NaYF}_{4}: \mathrm{Yb}, \mathrm{Er}$ 复合材料, 发现 $\mathrm{Ag}$ 纳米 线能够增强 $\mathrm{NaYF}_{4}: \mathrm{Yb}, \mathrm{Er}$ 纳米晶的上转换发光. $\mathrm{Liu}^{\text {等 }}{ }^{[24]}$ 通过两步法以柠檬酸钠为表面活性剂, 制备了 $\mathrm{Au} @ \beta-\mathrm{NaYF}_{4}: \mathrm{Yb}, \mathrm{Tm}$ 复合纳米粒子, 且 $\mathrm{Au}$ 修饰后上转 换发光明显增强. $\mathrm{Xu}$ 等 ${ }^{[25]}$ 用一种简便的两步种子介导 生长法, 首次制备了 $\mathrm{YVO}_{4}: \mathrm{Eu}^{3+} @ \mathrm{Ag}$ 核壳纳米混合溶 胶. 发现 $\mathrm{YVO}_{4}: \mathrm{Eu}^{3+}$ 表面的银纳米颗粒可以有效地阻止 $\mathrm{VO}_{4}^{3-}$ 和 $\mathrm{Eu}^{3+}$ 与溶剂的相互作用, 导致非辐射能量转移 (ET)的减少, 从而抑制了菼光猝灭. 在紫外区域有很强 的吸收, 在红外区域有很高的光致发光效率. Yuan 等 ${ }^{[26]}$ 制备了不同 $\mathrm{SiO}_{2}$ 层厚度的 $\mathrm{NaYF}_{4}: \mathrm{Yb}, \mathrm{Er} @ \mathrm{SiO}_{2} @ \mathrm{Ag}$ 核壳 纳米复合材料, 系统地研究了距离与金属增强上转换纳 米晶发光的关系. 发现金属和发光材料的间隔距离为
$10 \mathrm{~nm}$ 时出现最大上转换发光增强, 该纳米复合材料在 $\mathrm{B} 16 \mathrm{~F} 0$ 细胞中具有强的生物成像能力.

本工作利用直接沉淀法合成了以贵金属 $\mathrm{Ag}$ 纳米粒 子为核, $\mathrm{SiO}_{2}$ 为隔离层, 以稀土氟化物 $\mathrm{GdF}_{3}: \mathrm{Er}, \mathrm{Yb}$ 为壳 的核壳结构 $\mathrm{Ag} @ \mathrm{SiO}_{2} @ \mathrm{GdF}_{3}: \mathrm{Er}, \mathrm{Yb}$ 上转换发光纳米材 料, 将贵金属的等离子体共振效应和稀土离子的上转换 发光的优良性质相结合. 研究了贵金属 $\mathrm{Ag}$ 纳米粒子对 稀土氟化物上转换发光材料的发光强度的影响.

\section{2 结果与讨论}

\section{1 形成过程分析}

$\mathrm{AgNO}_{3}$ 在还原剂乙二醇作用下被还原为粒径可调 的 Ag 纳米颗粒. 通过改良后的 Stöber 方法对其表面包 覆上均匀的 $\mathrm{SiO}_{2}$ 层, 将包覆好的 $\mathrm{Ag} @ \mathrm{SiO}_{2}$ 纳米颗粒分 散于一定量的稀土硝酸盐和 $\mathrm{NH}_{4} \mathrm{~F}$ 的混合液中, 通过 $\mathrm{Ag} @ \mathrm{SiO}_{2}$ 表面的具有活性的 $\mathrm{Si}-\mathrm{OH}$ 基与稀土离子相互 作用, 将发光材料连接到 $\mathrm{Ag} @ \mathrm{SiO}_{2}$ 复合纳米粒子的表 面, 合成出 $\mathrm{Ag} @ \mathrm{SiO}_{2} @ \mathrm{GdF}_{3}: \mathrm{Er}, \mathrm{Yb}$ 复合纳米上转换发光 粒子. 形成过程示意图如图 1 所示.

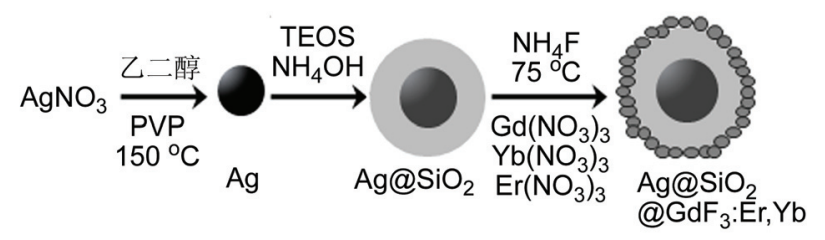

图1 $1 \mathrm{Ag} @ \mathrm{SiO}_{2} @ \mathrm{GdF}_{3}: \mathrm{Er}, \mathrm{Yb}$ 的制备过程示意图

Figure 1 Synthesis procedure scheme of $\mathrm{Ag} @ \mathrm{SiO}_{2} @ \mathrm{GdF}_{3}: \mathrm{Er}, \mathrm{Yb}$

\section{2 透射电镜(TEM)分析}

图 2 为 $\mathrm{Ag} @ \mathrm{SiO}_{2}$ 复合纳米粒子的 TEM 照片. 从照 片中可以清晰地看到 $\mathrm{Ag}$ 核外部包覆着不同厚度的 $\mathrm{SiO}_{2}$ 层, 且包覆均匀完全, 表面光滑, 为球形核壳结构. $\mathrm{Ag} @ \mathrm{SiO}_{2}$ 复合纳米粒子中, $\mathrm{Ag}$ 纳米粒子尺寸约为 $50 \mathrm{~nm}$, $\mathrm{Ag} @ \mathrm{SiO}_{2}$ 复合纳米粒子的尺寸约为 $70 \sim 110 \mathrm{~nm} . \mathrm{SiO}_{2}$ 层 厚度通过加入 $\mathrm{TEOS}$ 和 $\mathrm{NH}_{4} \mathrm{OH}$ 的量来调节, 分别为 10 $\mathrm{nm}$ (图 2a)、15 nm(图 2b) 和 $20 \mathrm{~nm}$ (图 2c) 左右.

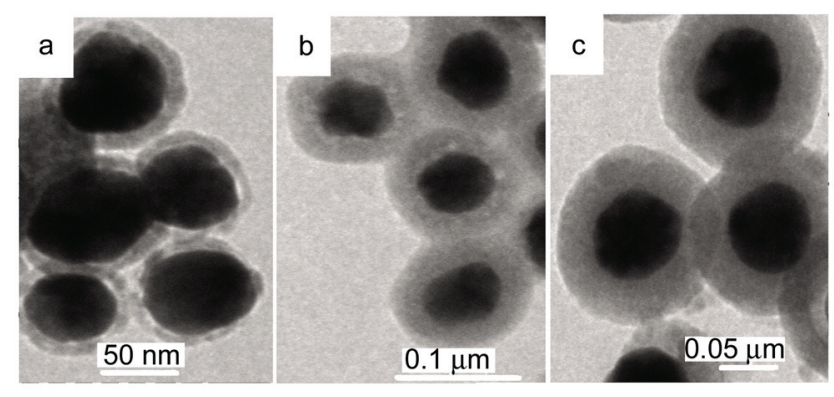

图 $2 \mathrm{Ag} @ \mathrm{SiO}_{2}$ 的 TEM 照片

Figure 2 TEM images of $\mathrm{Ag} @ \mathrm{SiO}_{2}$

(a) TEOS $0.1 \mathrm{~mL}, \mathrm{NH}_{4} \mathrm{OH} 10 \mathrm{~mL}$; (b) TEOS $0.15 \mathrm{~mL}, \mathrm{NH}_{4} \mathrm{OH} 15 \mathrm{~mL}$; (c) TEOS $0.2 \mathrm{~mL}, \mathrm{NH}_{4} \mathrm{OH} 20 \mathrm{~mL}$ 
图 3 为 $\mathrm{Ag} @ \mathrm{SiO}_{2} @ \mathrm{GdF}_{3}: \mathrm{Er}, \mathrm{Yb}$ 复合纳米发光粒子的 $\mathrm{TEM}$ 照片. 从照片中可看到，贵金属中心核与 $\mathrm{SiO}_{2}$ 层 以及稀土发光材料的外壳层之间有着明显的祄度对比, 表明 $\mathrm{Ag}$ 表面成功包覆 $\mathrm{SiO}_{2}$ 纳米层和 $\mathrm{GdF}_{3}: \mathrm{Er}, \mathrm{Yb}$ 纳米 发光粒子, 形成了核壳结构. $\mathrm{Ag} @ \mathrm{SiO}_{2} @ \mathrm{GdF}_{3}: \mathrm{Er}, \mathrm{Yb}$ 复 合纳米发光材料为球形结构, 复合后的粒径进一步增加, 约 80 120 nm, 且分散性良好. 从图 $3 \mathrm{e}$ 和 $3 \mathrm{f}$ 中可以看 出, 大量的 $\mathrm{GdF}_{3}: \mathrm{Er}, \mathrm{Yb}$ 纳米粒子吸附到 $\mathrm{SiO}_{2}$ 层上, 将其 空隙填充完全, 使得 $\mathrm{GdF}_{3}: \mathrm{Er}, \mathrm{Yb}$ 包覆层均匀、完全, 仍 为核壳结构. 而图 $3 \mathrm{a}, 3 \mathrm{~b}$ 和 $3 \mathrm{c}$ 中显示, 由于 $\mathrm{GdF}_{3}: \mathrm{Er}, \mathrm{Yb}$ 比例的减少, 其以小颗粒状态吸附于 $\mathrm{SiO}_{2}$ 的表层, 颗粒 尺寸约为 $7 \mathrm{~nm}$, 且无团聚现象发生, 整个产物粒子表面 包覆完全. 图 $3 \mathrm{~d}$ 为样品的高分辨 TEM 照片, 可以看出 $\mathrm{Ag}$ 球核与 $\mathrm{SiO}_{2}$ 层以及稀土壳层之间的祄度明显, 氟化 钝的晶面条纹间距为 $0.1481 \mathrm{~nm}$, 与 $\mathrm{GdF}_{3}(420)$ 晶面的晶 面间距 $0.1487 \mathrm{~nm}$ 相近, 内部银核的晶面条纹间距为 $0.1998 \mathrm{~nm}$, 对应于 $\mathrm{Ag}$ 的(200)晶面的晶面间距 0.2044 $\mathrm{nm}$ 相近, 证明在 $\mathrm{Ag}$ 的表面成功包覆上 $\mathrm{GdF}_{3}: \mathrm{Er}, \mathrm{Yb}$ 纳米 粒子, 合成出的产物具有核壳结构. 由图 a 内部的电子 衍射照片能够看到衍射环和衍射斑点，表明 $\mathrm{Ag} @ \mathrm{SiO}_{2} @ \mathrm{GdF}_{3}: \mathrm{Er}, \mathrm{Yb}$ 复合纳米发光粒子属于多晶结 构.
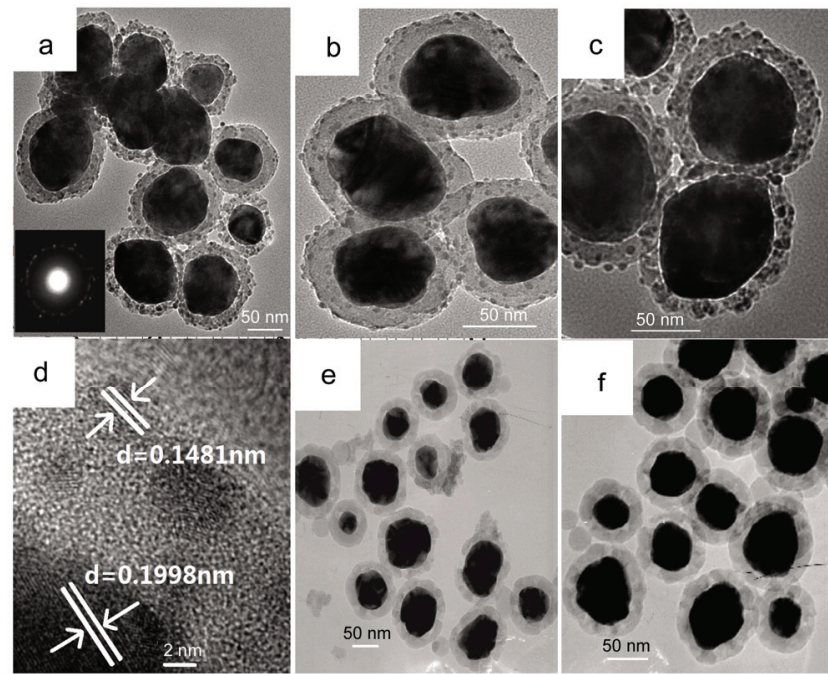

图 3 不同 $\mathrm{Ag} @ \mathrm{SiO}_{2}$ 与 $\mathrm{GdF}_{3}: \mathrm{Er}, \mathrm{Yb}$ 物质的量之比 $(\mathrm{a} \sim \mathrm{d}$ 为 $1: 1 ; \mathrm{e}, \mathrm{f}$ 为 $1: 5$ ) 的 $\mathrm{Ag} @ \mathrm{SiO}_{2} @ \mathrm{GdF}_{3}: \mathrm{Er}, \mathrm{Yb}$ 样品的 TEM 照片

Figure 3 TEM images of the $\mathrm{Ag} @ \mathrm{SiO}_{2} @ \mathrm{GdF}_{3}: \mathrm{Er}, \mathrm{Yb}$ samples with different molar ratio $(\mathrm{a} \sim \mathrm{d}$ is $1: 1$; e, f is $1: 5)$ of $\mathrm{Ag} @ \mathrm{SiO}_{2}$ and $\mathrm{GdF}_{3}: \mathrm{Er}, \mathrm{Yb}$

\section{$2.3 X$ 射线衍射(XRD)分析}

图 4 为 $\mathrm{Ag}, \mathrm{Ag} @, \mathrm{SiO}_{2}$ 以及不同 $\mathrm{SiO}_{2}$ 厚度的 $\mathrm{Ag} @$ $\mathrm{SiO}_{2} @ \mathrm{GdF}_{3}: \mathrm{Er}, \mathrm{Yb}$ 复合纳米粒子的 $\mathrm{XRD}$ 谱图. 图 4a 为 结晶良好的 $\mathrm{Ag}$ 单质. 图 $4 \mathrm{~b}$ 中包覆 $\mathrm{SiO}_{2}$ 后的样品除了单 质 $\mathrm{Ag}$ 的衍射峰外, 在 $20^{\circ}$ 附近有一宽的弥散的衍射峰, 认为是无定形 $\mathrm{SiO}_{2}$ 产生的. 图 $4 \mathrm{c} \sim 4 \mathrm{e}$ 所有衍射峰的位 置和相对强度与标准卡片 $\mathrm{Ag}(\mathrm{PDF} \# 04-0783)$ 以及 $\mathrm{GdF}_{3}$
(PDF\#49-1804)标准谱非常吻合, 并没有出现任何杂峰, 表明所合成的样品为纯相 $\mathrm{Ag}$ 和纯相 $\mathrm{GdF}_{3}$. 谱图中尖而 强的衍射峰进一步表明样品具有较好的结晶性. 同时, 随着 $\mathrm{SiO}_{2}$ 层厚度的增加, 对 $\mathrm{GdF}_{3}$ 和 $\mathrm{Ag}$ 的衍射峰的强度 的影响并不明显. 值得注意的是, 因为 $\mathrm{Er}^{3+}, \mathrm{Yb}^{3+}$ 是以 取代 $\mathrm{Gd}^{3+}$ 的方式进入到 $\mathrm{GdF}_{3}$ 晶格中的, 所以 $\mathrm{Er}^{3+}, \mathrm{Yb}^{3+}$ 的掺杂并不影响样品的晶体结构.

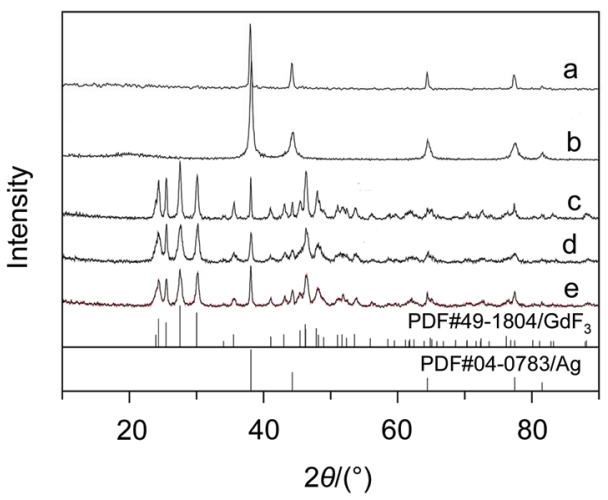

图 4 样品的 XRD 谱图

Figure 4 XRD patterns of the samples

(a) Ag; (b) $\mathrm{Ag} @ \mathrm{SiO}_{2}$; (c) $\mathrm{Ag} @ \mathrm{SiO}_{2}(20 \mathrm{~nm}) @ \mathrm{GdF}_{3}: \mathrm{Er}, \mathrm{Yb}$; (d) $\mathrm{Ag} @ \mathrm{SiO}_{2}(15$ $\mathrm{nm}) @ \mathrm{GdF}_{3}: \mathrm{Er}, \mathrm{Yb} ;(\mathrm{e}) \mathrm{Ag} @ \mathrm{SiO}_{2}(10 \mathrm{~nm}) @ \mathrm{GdF}_{3}: \mathrm{Er}, \mathrm{Yb}$

\section{4 紫外-可见吸收光谱(UV-Vis)分析}

图 5 为样品 $\mathrm{Ag}, \mathrm{Ag} @ \mathrm{SiO}_{2}$ 和不同氟化物厚度的 $\mathrm{Ag} @ \mathrm{SiO}_{2} @ \mathrm{GdF}_{3}: \mathrm{Er}, \mathrm{Yb}$ 复合纳米发光粒子的紫外-可见 吸收光谱. 从图中可以看出, 包覆 $\mathrm{SiO}_{2}$ 层后 $\mathrm{Ag}$ 的表面 等离子体共振吸收峰从 $429 \mathrm{~nm}$ 红移到了 $440 \mathrm{~nm}$. 在包 覆 $\mathrm{GdF}_{3}: \mathrm{Er}, \mathrm{Yb}$ 纳米粒子后, 吸收峰相对于 $\mathrm{Ag}$ 和 $\mathrm{Ag} @ \mathrm{SiO}_{2}$ 发生了进一步红移, 分别为 457, 466, $479 \mathrm{~nm}$, 且随着 $\mathrm{GdF}_{3}: \mathrm{Er}, \mathrm{Yb}$ 壳层厚度的增加, 红移程度越大. 原 因可能是 $\mathrm{GdF}_{3}: \mathrm{Er}, \mathrm{Yb}$ 和 $\mathrm{SiO}_{2}$ 成功包覆在 $\mathrm{Ag}$ 表层, 使 得 $\mathrm{Ag}$ 纳米粒子周围的局域折射率逐渐增强, 进而导致 $\mathrm{Ag}$ 纳米粒子的表面等离子体共振吸收峰发生了红移.

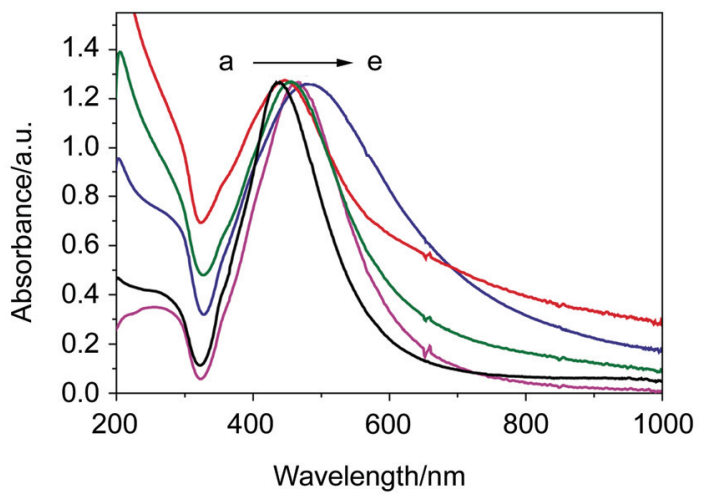

图 5 样品的紫外-可见吸收光谱图

Figure $5 \mathrm{UV}$-Vis spectra of the samples

$\mathrm{Ag}$ (a); $\mathrm{Ag} @ \mathrm{SiO}_{2}$ (b); the samples with different molar ratio of $\mathrm{Ag} @ \mathrm{SiO}_{2}$ and $\mathrm{GdF}_{3}: \mathrm{Er}, \mathrm{Yb}:$ (c) $1: 1$, (d) $1: 3$, (e) $1: 5$ 


\section{5 红外光谱(FTIR)分析}

图 6 分别给出了 $\mathrm{Ag} @ \mathrm{SiO}_{2}, \mathrm{Ag} @ \mathrm{SiO}_{2} @ \mathrm{GdF}_{3}: \mathrm{Er}, \mathrm{Yb}$ 和 $\mathrm{GdF}_{3}: \mathrm{Er}, \mathrm{Yb}$ 纳米粒子的红外光谱图. 由曲线 $\mathrm{a}$ 可见, 在 3442 和 $1637 \mathrm{~cm}^{-1}$ 附近有 $\mathrm{OH}$ 和 $\mathrm{H}_{2} \mathrm{O}$ 的振动峰; 1104, $802 \mathrm{~cm}^{-1}$ 附近为 $\mathrm{Si}-\mathrm{O}-\mathrm{Si}$ 的反对称与对称伸缩振动峰; $953 \mathrm{~cm}^{-1}$ 附近为 $\mathrm{Si}-\mathrm{OH}$ 的对称伸缩振动峰, 说明 $\mathrm{Ag}$ 纳米粒子表面有 $\mathrm{SiO}_{2}$ 的包覆, $\mathrm{SiO}_{2}$ 层的表面有大量的 $\mathrm{OH}$ 和 $\mathrm{H}_{2} \mathrm{O}$ 的存在, 有助于与贵金属离子发生键合作用 且易于稀土氟化物的包覆.

在曲线 b 中, 可以观察到 $3298,1630,1610 \mathrm{~cm}^{-1}$ 处 的吸收峰为 $\mathrm{OH}$ 的伸缩振动峰, 是样品吸收水分所致; $1432 \mathrm{~cm}^{-1}$ 处的振动吸收峰, 可能是表层吸附 $\mathrm{CO}_{2}$ 所致; $1110 \mathrm{~cm}^{-1}$ 附近为 $\mathrm{Si}-\mathrm{O}-\mathrm{Si}$ 的反对称伸缩振动峰, 同曲 线 $\mathrm{a}$ 相比, 此峰发生了位移并且强度有所减弱, 表明 $\mathrm{GdF}_{3}: \mathrm{Er}, \mathrm{Yb}$ 成功包覆在 $\mathrm{SiO}_{2}$ 表层.

由曲线 $\mathrm{c}$ 可见, 在 3387 和 $1630 \mathrm{~cm}^{-1}$ 附近有 $\mathrm{OH}$ 和 $\mathrm{H}_{2} \mathrm{O}$ 的振动峰; $1432 \mathrm{~cm}^{-1}$ 处的振动吸收峰, 可能是表层 吸附 $\mathrm{CO}_{2}$ 所致. 而无机物 $\mathrm{GdF}_{3}: \mathrm{Er}, \mathrm{Yb}$ 纳米发光粒子在红 外光谱中并没有明显的吸收峰, 这与氟化物在中红外区 没有吸收相一致 ${ }^{[27,28]}$.

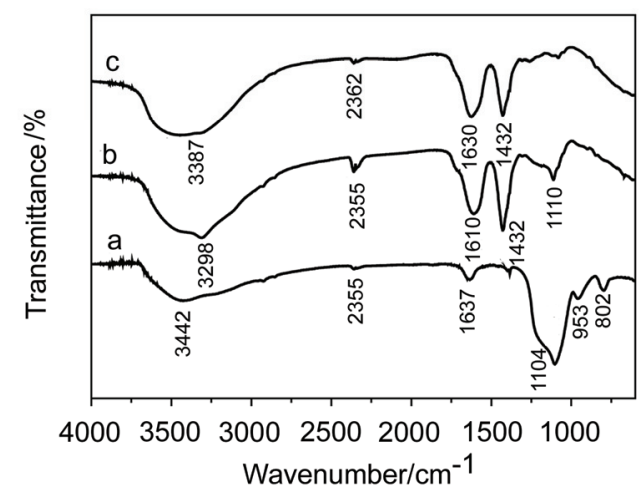

图 6 样品的红外吸收光谱图

Figure 6 FTIR spectra of the samples

(a) $\mathrm{Ag} @ \mathrm{SiO}_{2}$; (b) $\mathrm{Ag} @ \mathrm{SiO}_{2} @ \mathrm{GdF}_{3}: \mathrm{Er}, \mathrm{Yb}$; (c) $\mathrm{GdF}_{3}: \mathrm{Er}, \mathrm{Yb}$

\section{6 荧光光谱分析}

图 7,8 显示 $980 \mathrm{~nm}$ 激发下不同 $\mathrm{SiO}_{2}$ 包覆层厚度和 不同稀土发光材料加入量的 $\mathrm{Ag} @ \mathrm{SiO}_{2} @ \mathrm{GdF}_{3}: \mathrm{Er}, \mathrm{Yb}$ 和 $\mathrm{GdF}_{3}: \mathrm{Er}, \mathrm{Yb}$ 纳米粒子的上转换荧光光谱图. 从光谱图中 可以看出, 包覆前后, 样品的上转换光谱中的谱峰位置 基本一致, 而且相对于纯的 $\mathrm{GdF}_{3}: \mathrm{Yb}, \mathrm{Er}$ 发光强度有所增 强. 各谱图中均有三个比较明显的发射峰分别位于 520 , 540 和 $655 \mathrm{~nm}$, 其中 $520,540 \mathrm{~nm}$ 为绿光发射, 分别来自 于 $\mathrm{Er}^{3+}$ 离子的 ${ }^{2} \mathrm{H}_{11 / 2} \rightarrow{ }^{4} \mathrm{I}_{15 / 2}$ 和 ${ }^{4} \mathrm{~S}_{3 / 2} \rightarrow{ }^{4} \mathrm{I}_{15 / 2}$ 跃迁发射; 而 $655 \mathrm{~nm}$ 为红光发射, 来自于 $\mathrm{Er}^{3+}$ 离子的 ${ }^{4} \mathrm{~F}_{9 / 2} \rightarrow{ }^{4} \mathrm{I}_{15 / 2}$ 跃迁 发射.

由图 7 可知, $\mathrm{Ag} @ \mathrm{SiO}_{2} @ \mathrm{GdF}_{3}: \mathrm{Er}, \mathrm{Yb}$ 的上转换发光 强度比纯的 $\mathrm{GdF}_{3}: \mathrm{Yb}, \mathrm{Er}$ 有所增强, 且随着 $\mathrm{SiO}_{2}$ 包覆层
厚度的增加，上转换发光强度逐渐增强. 由于金属粒子 与荧光团之间距离的差异将导致荧光材料产生苂光发 射增强与减弱 ${ }^{[29,30]}$ : (1) $5 \mathrm{~nm}<d<15 \mathrm{~nm}$ ，金属表面等离 子体共振产生的局域场增强(LFE), 使苂光分子的激发 效率得到提高; (2) $15 \mathrm{~nm}<d<25 \mathrm{~nm}$, 金属纳米粒子的 表面等离子体共振发射(SPCE)增加苂光才的辐射衰减 率，降低了上转换材料到金属纳米粒子之间的无辐射能 量传递，使分子苂光强度增强. 我们合成的 $\mathrm{SiO}_{2}$ 层在 $10 \sim 20 \mathrm{~nm}$ 之间，在增强荧光的范围内，所以 $\mathrm{SiO}_{2}$ 层的 存在使得银对 $\mathrm{GdF}_{3}: \mathrm{Er}, \mathrm{Yb}$ 起到了苂光增强的作用，增强 机理图如图 8 所示.

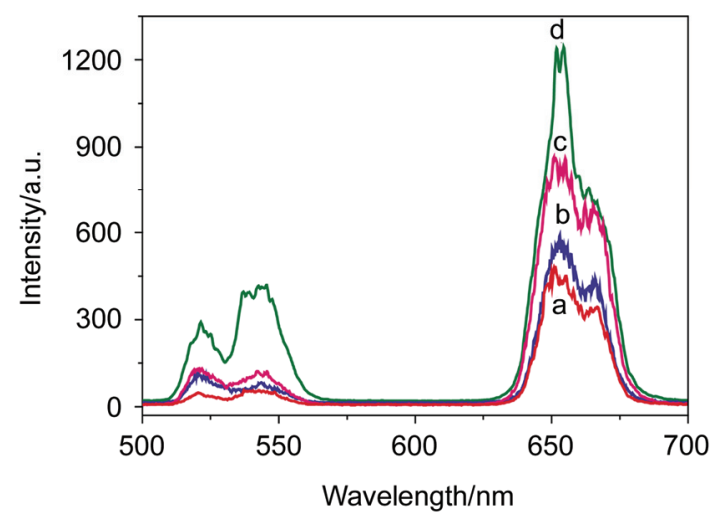

图 $7980 \mathrm{~nm}$ 激发下样品的上转换苂光光谱

Figure 7 Up-conversion spectra of the samples under $980 \mathrm{~nm}$ excitation (a) $\mathrm{GdF}_{3}: \mathrm{Er}, \mathrm{Yb}$; (b) $\mathrm{Ag} @ \mathrm{SiO}_{2}(10 \mathrm{~nm}) @ \mathrm{GdF}_{3}: \mathrm{Er}, \mathrm{Yb}$; (c) $\mathrm{Ag} @ \mathrm{SiO}_{2}(15$ nm)@GdF $:$ Er,Yb; (d) Ag@ $\mathrm{SiO}_{2}\left(20\right.$ nm)@ $\mathrm{GdF}_{3}: \mathrm{Er}, \mathrm{Yb}$

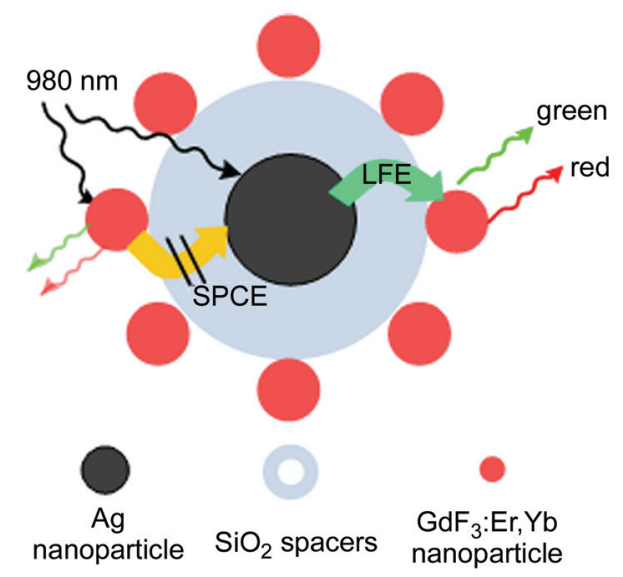

图 8 复合粒子苂光增强的机理图

Figure 8 Mechanics scheme of luminescence enhancement of composite particles

图 9 是在 $\mathrm{SiO}_{2}$ 层不变的情况下, 增加 $\mathrm{GdF}_{3}: \mathrm{Yb}, \mathrm{Er}$ 壳层的比例, 得到样品的苂光光谱图. 由图 9 可看出, 在 $\mathrm{SiO}_{2}$ 层不变的情况下, 增加 $\mathrm{GdF}_{3}: \mathrm{Yb}, \mathrm{Er}$ 壳层的比例, 上转换发光强度也逐渐增强. 综上所述, $\mathrm{Ag}$ 核的存在, 对上转换发光强度得到了增强, 随着 $\mathrm{SiO}_{2}$ 隔离层厚度 的增加以及稀土发光材料比例的增加, 发光增强明显. 


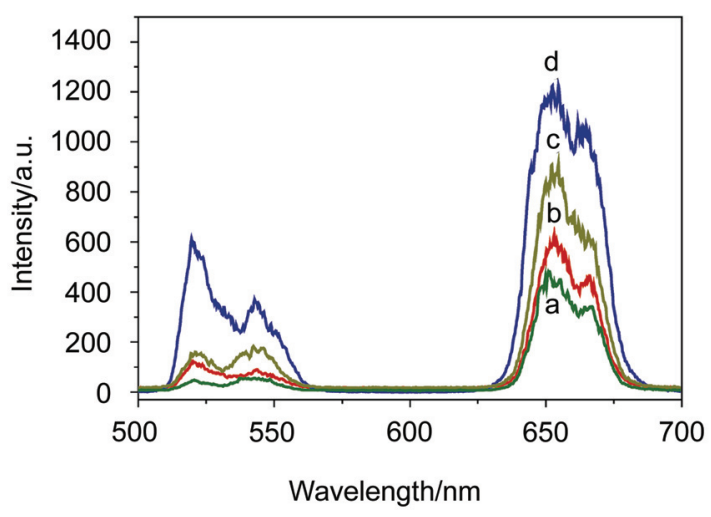

图 $9980 \mathrm{~nm}$ 激发下样品的上转换荧光光谱

Figure 9 UC spectra of the samples under $980 \mathrm{~nm}$ excitation (a) $\mathrm{Ag} \mathrm{GdF}$ : $\mathrm{Er}, \mathrm{Yb}$. The samples with different molar ratio of $\mathrm{Ag} @ \mathrm{SiO}_{2}$ and $\mathrm{GdF}_{3}: \mathrm{Er}, \mathrm{Yb}:(\mathrm{b}) 1: 1$, (c) $1: 3$, (d) $1: 5$

图 10 所示为核壳结构样品红光和绿光跃迁发射的 上转换发光强度与激发功率的双对数关系图. 在上转换 过程中, 上转换发光强度 $(I)$ 与激发功率 $(P)$ 具有如下关 系: $I \propto P^{n}$, 其中 $n$ 代表发射一个上转换光子所需要的激 发光子数. 由图可见, 红光和绿光跃迁的 $n$ 值分别为 2.10 和 2.18 , 所以, 红光和绿光发射均为双光子过程.

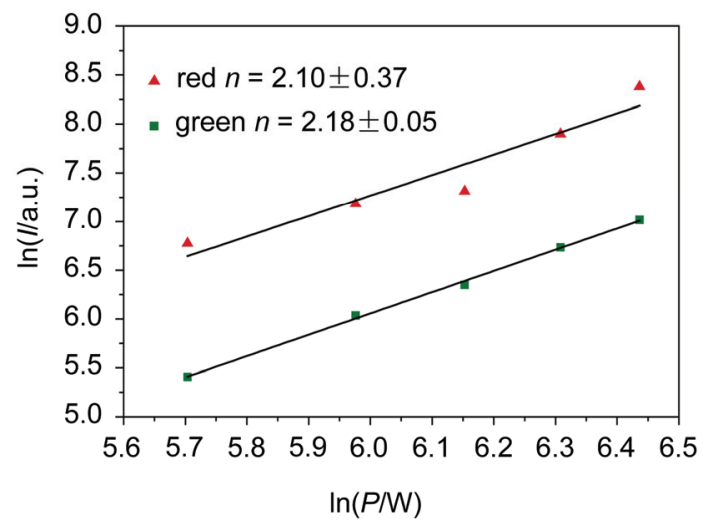

图 10 上转换发光强度 $(I)$ 与激发功率 $(P)$ 的双对数图

Figure 10 Plots of logarithm intensity of the up-conversion emissions ( $I$ ) versus logarithm pumped power of diode laser $(P)$

\section{3 结论}

本文采用液相法制备了 $50 \mathrm{~nm}$ 的单质 $\mathrm{Ag}$ 纳米粒子, 再利用直接沉淀法成功制备了不同 $\mathrm{SiO}_{2}$ 包覆层厚度和 不同稀土发光材料比例的 $\mathrm{Ag} @ \mathrm{SiO}_{2} @ \mathrm{GdF}_{3}: \mathrm{Er}^{3+}, \mathrm{Yb}^{3+}$ 核 壳结构纳米上转换发光粒子. 研究结果表明: 得到的复 合纳米粒子为球形核壳结构, $\mathrm{GdF}_{3}: \mathrm{Er}^{3+}, \mathrm{Yb}^{3+}$ 纳米粒子 均匀的包覆在 $\mathrm{Ag} @ \mathrm{SiO}_{2}$ 核表面. 包覆后的复合纳米粒 子的紫外-可见吸收峰相对于 $\mathrm{Ag}$ 核发生了进一步红移. 在 $980 \mathrm{~nm}$ 激发下该复合纳米粒子具有良好的上转换发 光性能, 发光强度比纯的 $\mathrm{GdF}_{3}: \mathrm{Er}, \mathrm{Yb}$ 明显增强, 表明 $\mathrm{SiO}_{2}$ 层的存在使得 $\mathrm{Ag}$ 纳米粒子对外层 $\mathrm{GdF}_{3}: \mathrm{Er}, \mathrm{Yb}$ 的发 光起到了增强的作用. 本工作为等离子体共振对稀土发
光材料发光作用机理的研究方面提供了一定的实验依 据. 此核壳结构在生物医学领域有着潜在的应用前景.

\section{4 实验部分}

\section{1 实验试剂}

氧化钝(纯度： $99.99 \%$, 上海跃龙有色金属有限公 司), 氧化镱 ( $\geqslant 99.9 \%$, 国药集团化学试剂有限公司), 氧化铒 $(\geqslant 99.9 \%$, 国药集团化学试剂有限公司), 氟化铵 (96.0\%, 天津市光复精细化工研究所), 硝酸银(纯度: $\geqslant 99.8 \%$, 国药集团化学试剂有限公司), 乙二醇(纯度: $\geqslant 99.7 \%$, 天津市富宇精细化工有限公司), 聚乙烯吡咯 烷酮(K30) (PVP, 天津市博迪化工有限公司), 正硅酸乙 酯 (TEOS, 纯度 $\geqslant 28.0 \%$, 北京化工厂), 氨水(纯度： $25 \% \sim 28 \%$, 北京化工厂), 异丙醇(纯度： $\geqslant 99.7 \%$, 天 津市天泰精细化学品有限公司), 硝酸(纯度: $65 \% \sim 68 \%$, 北京化工厂), 无水乙醇 ( $\geqslant 99.7 \%$, 北京化工厂).

\section{2 制备过程}

\subsubsection{Ag 纳米粒子的制备}

根据文献[31]所述的方法, 具体步骤为: 称取 $0.2 \mathrm{~g}$ 的 $\mathrm{AgNO}_{3}$ 和 $1 \mathrm{~g}$ 的 PVP $(K=30)$ 溶解于 $80 \mathrm{~mL}$ 的乙二醇 中, 搅拌 $20 \mathrm{~min}$ 使 PVP 充分溶解. 然后将混合物以 10 ${ }^{\circ} \mathrm{C} / \mathrm{min}$ 的速度加热到 $130{ }^{\circ} \mathrm{C}$, 此温度下恒温 $1 \mathrm{~h}$. 之后 加入大量的丙酩进行离心 $(8000 \mathrm{r} / \mathrm{min}, 5 \mathrm{~min})$, 用去离子 水和乙醇洗涤三次, 离心出的沉淀物保存在乙醇当中, 以备后用。

\subsection{2 $\mathrm{Ag} @ \mathrm{SiO}_{2}$ 复合纳米粒子的制备}

将制备出的单质 $\mathrm{Ag}$ 溶于 $120 \mathrm{~mL}$ 异丙醇中, 超声 $30 \mathrm{~min}$, 之后加入一定量的 $\mathrm{NH}_{4} \mathrm{OH}$ 和蒸馏水, 然后快速 的分别加入 $0.1,0.15$ 和 $0.2 \mathrm{~mL}$ 的 TEOS, 室温下搅拌 $4 \mathrm{~h}$, 将反应后的溶胶进行离心 $(8000 \mathrm{r} / \mathrm{min}, 10 \mathrm{~min})$, 用去离 子水和乙醇分别洗涤三次, 保存在乙醇当中备用.

\subsection{3 $\mathrm{Ag} @ \mathrm{SiO}_{2} @ \mathrm{GdF}_{3}: \mathrm{Er}, \mathrm{Yb}$ 复合纳米发光粒子的制 备}

先配制 $0.2 \mathrm{~mol} / \mathrm{L}$ 的 $\mathrm{Gd}\left(\mathrm{NO}_{3}\right)_{3}, 0.2 \mathrm{~mol} / \mathrm{L}$ 的 $\mathrm{Er}\left(\mathrm{NO}_{3}\right)_{3}$, $0.2 \mathrm{~mol} / \mathrm{L}$ 的 $\mathrm{Yb}\left(\mathrm{NO}_{3}\right)_{3}$ 以及 $0.6 \mathrm{~mol} / \mathrm{L}$ 的 $\mathrm{NH}_{4} \mathrm{~F}$ 溶液, 以 备实验使用. 在摚拌情况下将制备出的 $\mathrm{Ag} @ \mathrm{SiO}_{2}$ 分散 于 $3.3 \mathrm{~mL} \mathrm{Gd}\left(\mathrm{NO}_{3}\right)_{3}, 0.1 \mathrm{~mL} \mathrm{Er}\left(\mathrm{NO}_{3}\right)_{3}, 1 \mathrm{~mL} \mathrm{Yb}\left(\mathrm{NO}_{3}\right)_{3}$ 以 及去离子水的混合溶液中, 形成混浊溶液, 摚拌 $30 \mathrm{~min}$. 再加入 $10 \mathrm{~mL}$ 的 $\mathrm{NH}_{4} \mathrm{~F}$ 溶液, 继续搅拌 $10 \mathrm{~min}$, 转移至 水浴锅中于 $75{ }^{\circ} \mathrm{C}$ 下搅拌 $2 \mathrm{~h}$ 后得棕黑色沉淀, 离心分 离(8000 r/min, $5 \mathrm{~min})$, 用去离子水和乙醇洗涤三次, 于 $80{ }^{\circ} \mathrm{C}$ 下干燥, 得棕黑色粉末.

\section{3 表征方法}

采用德国 Bruker D\&FOCUS 多晶粉末 X 射线衍射 (XRD)仪进行物相分析, 其 $\mathrm{X}$ 射线源为 $\mathrm{Cu} \mathrm{K \alpha} \alpha_{1}, \lambda=$ $1.54056 \AA$, 起始角度为 $20^{\circ} \sim 90^{\circ}$, 步长为 $0.2^{\circ}$, 扫描速 率为 $0.1\left(^{\circ}\right) / \mathrm{s}$; 日本 JEOL 公司生产的 JEM-2010 型透射 
电镜(TEM)观察产物的形貌及其尺寸; 采用德国 Bruker Optics 公司 BRUKEU Vertex 70 红外光谱仪进行样品的 FTIR 分析. 粉末试样采用 $\mathrm{KBr}$ 压片法, 其扫描范围为 $4000 \sim 400 \mathrm{~cm}^{-1}$; 采用苏州奥科计量仪器有限公司岛津 UV-1240 型紫外可见分光光度计进行样品的紫外可见 吸收光谱测试; 采用日立 F-7000 型苂光光谱仪以 MDL-W980 nm 半导体激光器激发, 功率为 $1 \mathrm{~W}$, 对样 品的上转换发光进行测试.

\section{References}

[1] Liu, C. Y.; Gao, Z. Y.; Zeng, J. F.; Hou, Y.; Fang, F.; Li, Y. L.; Qiao, R. R.; Shen, L.; Lei, H.; Yang, W. S.; Gao, M. Y. ACS Nano 2013, 7, 7227.

[2] Idris, N. M.; Li, Z. Q.; Ye, L. J. Biomaterials 2009, 30, 5104.

[3] Liu, Y. S.; Tu, D. T.; Zhu, H. M.; Chen, X. Y. Chem. Soc. Rev. 2013, 42, 6924.

[4] Li, C. X.; Lin, J. J. Mater. Chem. 2010, 20, 6831.

[5] Gu, Z. G.; Wang, B. X.; Pang, C. Y.; Zhou, W.; Li, Z. J. Acta Chim. Sinica 2012, 70, 2501. (顾志国, 王宝祥, 庞春燕, 周文, 李在均, 化学学报, 2012, 70, 2501.)

[6] Wang, Y. H.; Wang, J. X.; Dong, X. T.; Yu, W. S.; Liu, G. X. Acta Chim. Sinica 2012, 70, 1576. (王莹熇, 王进贤, 董相廷, 于文生, 刘桂霞, 化学学报, 2012, 70, 1576.)

[7] Yue, D.; Li, C. Y.; Zhang, X. L.; Chang, J. Z.; Wang, Z. L. Acta Chim. Sinica 2012, 70, 1812. (岳丹, 李春阳, 鲁伟, 张新否, 常加 忠, 王振领, 化学学报, 2012, 70(17), 1812.)

[8] Wang, X.; Han, Y. D.; Hao, S. Q.; Yu, J. H.; Xu, R. R. Acta Chim. Sinica 2012, 70, 1496. (王㬢, 韩义德, 郝素琴, 于吉红, 徐如人, 化学学报, 2012, 70, 1496.)

[9] Gai, S. L.; Yang, P. P.; Li, C. X.; Wang, W. X.; Dai, Y. L.; Niu, N.; Lin, J. Adv. Funct. Mater. 2010, 20, 1166.
[10] Zhou, J.; Liu, Z.; Fu, Y. L. Chem. Soc. Rev. 2012, 41, 1323

[11] Wang, F.; Liu, X. G. J. Am. Chem. Soc. 2008, 130, 5642.

[12] Huang, Q. M.; Yu, H.; Zhang, X. Q.; Yu, J. C. Acta Chim. Sinica 2013, 71, 1071. (黄清明, 俞瀚, 张新奇, 俞建长, 化学学报, 2013 $71,1071$.

[13] Cheng, L.; Wang, C.; Liu, Z. Nanoscale 2013, 5, 23.

[14] Feng, W.; Han, C. M.; Li, F. Y. Adv. Mater. 2013, 25, 5287

[15] Gu, Z. J.; Yan, L.; Tian, G.; Li, S. J.; Chai, Z. F.; Zhao, Y. L. Adv. Mater. 2013, 25, 3758.

[16] Wiley, B. J.; Chen, Y.; McLellan, J. Nano Lett. 2007, 7, 1032.

[17] Zhang, Q.; Li, W.; Moran, C.; Zeng, J.; Chen, J.; Wen, L. P.; Xia, Y. J. Am. Chem. Soc. 2010, 132, 11372.

[18] Lee, Y. W.; Kim, M.; Kim, Z. H.; Han, S. W. J. Am. Chem. Soc. 2009, 131, 17036.

[19] Sobha, K.; Surendranath, K.; Meena, V.; Jwala, K.; Swetha, N.; Biotechnol. Mol. Biol. Rev. 2010, 51, 1 .

[20] Zhu, X. J.; Zhou, J.; Chen, M.; Shi, M.; Feng, W.; Li, F. Y. Biomaterials 2012, 33, 4618.

[21] Aslan, K.; Wu, M.; Lakowicz, J. R.; Geddes, C. D. J. Am. Chem. Soc. 2007, 129, 1524

[22] Min, Y. L.; Wan, Y.; Yu, S. H. Solid State Sci. 2009, 11, 96.

[23] Feng, W.; Sun, L. D.; Yan, C. H. Chem. Commun. 2009, 4393.

[24] Liu, N.; Qin, W. P.; Qin, G. S.; Jiang, T.; Zhao, D. Chem. Commun. 2011, 7671.

[25] Xu, W.; Bai, X.; Xu, S.; Zhu, Y. S.; Xia, L.; Song, H. W. RSC Adv. 2012, 2, 2047.

[26] Yuan, P. Y.; Lee, Y. H.; Gnanasammandhan, M. K.; Guan, Z. P.; Zhang, Y.; Xu, Q. H. Nanoscale 2012, 4, 5132.

[27] Darbandi, M.; Nann, T. Chem. Commun. 2006, 776.

[28] Rahman, P.; Green, M. Nanoscale 2009, 1, 214.

[29] Lakowicz, J. R. Anal. Biochem. 2001, $298,1$.

[30] He, X.; Zhang, M.; Feng, J. Y.; Song, M. X.; Zhao, X. J. Rare Earth Mater. Eng. 2011, 40, 559. (何釒鍂, 张梅, 冯晋阳, 宋明霞, 赵修 建, 稀有金属材料与工程, 2011, 40, 559.)

[31] Zhang, F.; Braun, G. B.; Shi, Y. F.; Zhang, Y. C.; Sun, X. H.; Reich, N. O.; Zhao, D. Y.; Stucky, G. J. Am. Chem. Soc. 2010, 132, 2850.

(Zhao, C.) 